[0212-7199 (2008) 25: 5; pp 213-221] ANALES DE MEDICINA INTERNA Copyright (C) 2008 ARAN EDICIONES, S.L.

AN. MED. INTERNA (Madrid) Vol. 25, N. ${ }^{\circ}$ 5, pp. 213-221, 2008

\section{Perfil de eficiencia en las derivaciones neurológicas efectuadas a los especialistas de referencia: utilización del sistema de casuística Ajusted Clinical Groups}

A. SICRAS-MAINAR, R. NAVARRO-ARTIEDA ${ }^{1}$

En representación del Grupo de Estudio ACG-CAT formado por: J. Codes-Marco, X. Frías-Garrido, R. Llausí-Sellés, J. R. Llopart-López, I. Ruano-Ruano, J. Serrat-Tarrés y S. Velasco-Velasco

Badalona Serveis Assistencials S.A. ${ }^{I}$ Servicio de Documentación Médica. Hospital Germans Trías i Pujol. Badalona, Barcelona
EFFICIENCY PROFILE IN THE NEUROLOGICAL REFERRALS EFFECTUATE REFERENCE SPECIALISTS: USE CASE-MIX SYSTEM ADJUSTED CLINICAL GROUPS

\section{RESUMEN}

Objetivos: Determinar la tasa de derivación general y neurológica por centros y los índices de eficiencia ajustados, mediante la aplicación retrospectiva de los Adjusted Clinical Groups (ACG) en el ámbito de la atención primaria.

Pacientes y métodos: Diseño de estudio retrospectivo y multicéntrico. Se incluyeron todos los pacientes atendidos en cinco equipos de atención primaria (EAP) durante el año 2006. Las principales medidas fueron: parámetros generales, edad, género, variables dependientes (visitas y episodios) y comorbilidades de cada paciente, agrupadas por ACG. La tasa de derivación fue definida como el cociente entre el número de derivaciones entre las visitas realizadas. El índice de eficiencia (IE) fue establecido por estandarización indirecta dividiendo las derivaciones observadas entre las esperadas. Significación estadística, $\mathrm{p}<0,05$.

Resultados: Los pacientes estudiados fueron 80.775 (uso: $72,4 \%$ ), $4,8 \pm 3,5$ episodios y 7,9 $\pm 8,2$ visitas/pacientes/año. El porcentaje de visitas con derivación fue del 9,0\% (intervalos de confianza [CI]: 8,89,2 ); edad 44,8 $\pm 22,8$ años (mujeres: $54,6 \%$ ), $\mathrm{p}=0,000$. El promedio de derivaciones fue de 70,5 por 100 pacientes atendidos/año $(\mathrm{p}=0,000)$. Del total de derivaciones un $2,5 \%$ fueron neurológicas, siendo pacientes de edad avanzada, con predominio de mujeres y la cefalea/migraña el principal motivo de consulta. Las visitas y los episodios explican un $43,2-73,9 \%$ respectivamente $(\mathrm{p}=0,000)$, el poder explicativo de la variabilidad de la clasificación fue del $46,3 \%(\mathrm{p}=0,0001)$ y las derivaciones del $20,1 \%$. Los IE por centro fueron: 0,97 (IC: $0,77-1,18$ ), 0,79 (IC: $0,57-$ $1,01$ ), 0,88 (IC: $0,62-1,14), 1,29$ (IC: $0,94-1,65)$ y 0,91 (IC: $0,58-1,25), p$ $=0,023$ (medicina de familia) y 0,90 (IC: $0,47-1,33$ ), 0,78 (IC: $0,35-$ $1,21), 0,93$ (IC: $0,43-1,44), 1,21$ (IC: $0,60-1,82$ ) y 0,97 (IC: $0,39-1,56), p$ $=0,031$ (pediatría); respectivamente.

Conclusiones: La morbilidad ajustada por ACG explica una parte importante de la variabilidad de las derivaciones. Las derivaciones a la especialidad de neurología fueron un bajo porcentaje del total. Los resultados del estudio deben de interpretarse con prudencia, incluso después del ajuste por edad, género y morbilidad. De confirmarse los resultados posibilitarían una mejora en la medida de las derivaciones en los EAP.

PALABRAS CLAVE: Adjusted Clinical Groups. Derivaciones. Atención primaria. Neurología. Eficiencia. Uso de recursos. Gestión clínica.
ABSTRACT

Objectives: To determine the general referral and neurological rate per center and the adjusted efficiency indexes, through the retrospective implementation of the Adjusted Clinical Groups (ACG) in a primary care setting.

Patients and methods: To design multicenter retrospective study. Attended patients by five primary care teams (PCT) during the year 2006 were included. The main measurements were general parameters, age, gender, dependent (visits and episodes) and morbidity of each patient relative to each ACG. The referral rate was defined as the quotient between the number of referrals and the visits made. Efficiency Index (EI) was established dividing the observed by the expected referrals obtained by indirect standardization. Statistical significance, $p<0.05$.

Results: Studied patients 80775 (use: $72.4 \%$ ), $4.8 \pm 3.5$ episodes and $7.9 \pm 8.2$ visits/patient/year. Percentage of visits with a referral was 9.0\% (confiance interval [CI]: 8.8-9.2); age: $44.8 \pm 22.8$ years (women: $54.6 \%), p=0.000$. The average of referrals was of 70.5 per 100 attended-patients/year $(p=0.000) 2.5 \%$ referrals of the total were made to the neurological, being patient of greater age, with predominance of women and displaying the head pain/migraine as main consultation reason. Visits and episodes explain $43.2 \%-73.9 \%$ respectively $(p=0.000)$, the explanatory power of the classification's variability was of $46.3 \%(p=$ 0.0001 ) and the referral 20.1\%. EI per center were: 0.97 (CI: 0.77-1.18), 0.79 (CI: 0.57-1.01), 0.88 (CI: 0.62-1.14), 1.29 (CI: 0.94-1.65) and 0.91 (CI: 0.58-1.25), $p=0.023$ (family practice) and 0.90 (CI: 0.47-1.33), 0.78 (CI: 0.35-1.21), 0.93 (CI: 0.43-1.44), 1.21 (CI: 0.60-1.82) and 0.97 (CI: 0.39-1.56), $p=0.031$ (pediatrics); respectively.

Conclusions: Adjusted morbidity by ACG explains an important part of the referrals variability. A low percentage was derived to neurology. The study results must be interpreted cautiously even after adjustment by age, gender and morbidity. Should the results be confirmed it would allow an improvement in the measurement of referrals for clinical management in the PCT.

KEY WORDS: Adjusted Clinical Groups. Referrals. Primary Care. Neurology. Efficiency. Use resources. Clinical management.

Sicras-Mainar A, Navarro-Artieda R. Perfil de eficiencia en las derivaciones neurológicas efectuadas a los especialistas de referencia: utilización del sistema de casuística Ajusted Clinical Groups. An Med Interna (Madrid) 2008; 25: 213-221. 


\section{INTRODUCCIÓN}

Los sistemas de clasificación de pacientes en atención primaria no han sido empleados de una forma generalizada ni siquiera en EE.UU., su principal lugar de origen $(1,2)$. Siguiendo la tendencia actual en separar financiación, compra y provisión de servicios, se requieren instrumentos más precisos de evaluación y medida de la actividad asistencial (3). Los Ajusted Clinical Groups (ACG) fueron desarrollados por Starfield y Weiner y su principal objetivo consiste en medir la carga de morbilidad en poblaciones, basándose en un similar consumo de recursos $(4,5)$. Constituyen una de las posibles metodologías de ajuste (Fig. 1), junto con las Hierarchical Coexisting Conditions o los Clinical Risk Groups, que se pueden utilizar para evaluar de una forma más precisa y equitativa, la financiación de los planes de salud de la administración o valorar la eficiencia en la utilización de los recursos sanitarios $(2,5-7)$.

Los trastornos neurológicos, en general, son un conjunto de enfermedades que afectan a las personas jóvenes y de forma más relevante a las de mayor edad. Son uno de los grupos de enfermedades que generan más coste, no sólo para el sistema sanitario (costes directos), sino para toda la sociedad en general (costes indirectos), siendo frecuentemente atendidos en los centros de atención primaria (8). En este aspecto, el acto de la derivación consiste en una transferencia total o parcial del cuidado del paciente, desde los equipos de atención primaria (EAP) a la atención especializada, siendo la segunda causa del consumo de recursos en la cuenta de pérdidas y ganancias (explotación) de los centros, después de la prescripción farmacéutica (3). Por tanto, estamos ante un aspecto de elevada magnitud en el sistema sanitario, donde el modelo asistencial alcanzado refuerza una atención de continuidad asistencial con criterios de eficiencia y capacidad resolutiva (9-13).

En la bibliografía disponible se encuentran numerosas evidencias relativas a la variabilidad en la práctica clínica de las tasas de derivación (entre 1 y $69 \%$ por visitas) $(8,14-17)$. Estudios poblacionales demuestran la existencia de diversos factores relacionados: características sociodemográficas de la población (patrón de morbilidad, áreas rurales o urbanas, etc.), del propio sistema sanitario (oferta de servicios, accesibilidad geográfica, coordinación entre los diferentes niveles asistenciales, adecuación de los protocolos de derivación, etc.) o factores dependientes de los profesionales (formación y experiencia, estilos de práctica, etc.), por citar algunos ejemplos $(10,18-21)$. El objeto del estudio fue determinar la tasa de derivación general y específica a la especialidad de neurología por centros y la obtención de los índices de eficiencia ajustados (casuística), mediante la aplicación retrospectiva de los ACG desde la atención primaria a la especializada en un ámbito poblacional español.

\section{PACIENTES Y MÉTODOS}

La población de estudio estuvo formada por personas de ambos sexos de cinco EAP reformados gestionados por Bada-

\begin{tabular}{|c|c|c|c|}
\hline Descripción & Variables & Codificación & Unidad de análisis \\
\hline $\begin{array}{l}\text { Diagnosis Clusters } \\
\text { (DC) }\end{array}$ & Diagnóstico principal & $\begin{array}{l}\text { CIE-9-MC } \\
\text { CIAP-2 } \\
\text { CIPSAP-2 }\end{array}$ & Diagnóstico/consulta \\
\hline $\begin{array}{l}\text { Ambulatory Visit Groups } \\
\text { (AVG) }\end{array}$ & $\begin{array}{l}\text { Diagnóstico principal } \\
\text { Procedimientos } \\
\text { Edad/Sexo } \\
\text { Relación con el paciente } \\
\text { Seguimiento } \\
\text { Diagnósticos secundarios }\end{array}$ & $\begin{array}{l}\text { CIE-9-MC } \\
\text { CPT-4 }\end{array}$ & Consulta/visita \\
\hline $\begin{array}{l}\text { Ambulatory Patient Groups } \\
\text { (APG) }\end{array}$ & $\begin{array}{l}\text { Diagnósticos } \\
\text { Procedimientos } \\
\text { Edad/Sexo }\end{array}$ & $\begin{array}{l}\text { CIE-9-MC } \\
\text { CPT-4 }\end{array}$ & Consulta/visita \\
\hline $\begin{array}{l}\text { Products of Ambulatory Care } \\
\text { (PAC) }\end{array}$ & $\begin{array}{l}\text { Diagnósticos } \\
\text { Edad/Sexo } \\
\text { Exámenes complementarios } \\
\text { Tipo de estructura } \\
\text { Tipo de producto } \\
\text { Relación con el paciente } \\
\text { Medicamentos }\end{array}$ & $\begin{array}{l}\text { CIE-9-MC } \\
\text { CPT-4 }\end{array}$ & Consulta/visita \\
\hline $\begin{array}{l}\text { Ambulatory Care Groups } \\
\text { (ACG) }\end{array}$ & $\begin{array}{l}\text { Diagnósticos } \\
\text { Edad } \\
\text { Sexo }\end{array}$ & CIE-9-MC & Paciente \\
\hline
\end{tabular}

CIE-9-MC: Clasificación Internacional de Enfermedades, 9ª Revisión, Modificación Clínica. CIAP-2: Clasificación Internacional de Atención Primaria. CIPSAP-2: Clasificación Internacional de los Problemas de Salud en Atención Primaria. CPT-4: Physicians' Current Procedural Terminology, $4^{a}{ }^{2}$ edición.

Fig. 1. Distribución de las variables independientes, codificación y unidad de análisis utilizadas en los principales sistemas de clasificación de pacientes ambulatorios. 
lona Serveis Assistencials SA, que da cobertura a una población urbana de referencia de unos 110.440 habitantes (un 15,9\% mayores de 64 años). La población asignada es en su mayoría urbana, de nivel socioeconómico medio-bajo, con predominio industrial. El tipo de organización es de carácter reformado, con titularidad pública y provisión de servicios privada (concertados con el CatSalut). La organización cuenta con una dotación de personal, política de formación, modelo organizativo y cartera de servicios similar a la mayoría de EAP de Cataluña; con un modelo de gestión descentralizada y servicios estructurales únicos. Se realizó un estudio transversal, multicéntrico realizado a partir de la revisión retrospectiva de los registros médicos de pacientes atendidos durante el año 2006 (pediátricos y de medicina de familia). Fueron excluidos los sujetos trasladados, los fallecidos y los que no acudieron durante el periodo de estudio.

Se contabilizaron las derivaciones efectuadas a los especialistas de referencia mediante la tasa de derivación (TD), definida como el cociente entre el número de derivaciones entre las visitas realizadas por 100, durante el año de estudio. De cada derivación se obtuvo el centro receptor (propia/distinta organización), prioridad (normal/preferente-urgente), especialidad emisora (servicios de medicina de familia/pediatría) y receptora (quirúrgicas/médicas; y por las diferentes especialidades), así como una serie de indicadores/parámetros de planificación, descritos en la tabla I. Además se obtuvo información de las siguientes variables: a) universales: edad y sexo; b) dependientes: visitas y episodios; y c) de casuística o comorbilidad. Se utilizó la variable edad para agrupar a los pacientes por servicios asistenciales, de este modo, los rangos de edad de 0 a 14 años agrupan a los pacientes de Pediatría, y los mayores de 14 años, a los de Medicina de Familia. Algunas derivaciones minoritarias efectuadas por otros profesionales (odontología, trabajador social, enfermería, etc.) se asignaron a estos dos servicios.

Se consideró el episodio como un proceso de atención de una enfermedad o una demanda explícita equivalente al diagnóstico, determinado según la Clasificación Internacional de la Atención Primaria (CIAP-2) (22). Los episodios seleccionados, no repetidos, en población atendida, se contabilizaron por las fechas de registro en el curso clínico de las historias clínicas para cada episodio/motivo de consulta con independencia de la fecha de apertura del diagnóstico (carga de morbilidad/paciente). Se realizó una conversión (mapping) de la CIAP a la Clasificación Internacional de Enfermedades (CIE9-MC); para su confección se constituyó un grupo de trabajo formado por cinco profesionales (un documentalista, dos médicos clínicos y dos técnicos consultores). Los criterios seguidos fueron diferentes según se estableciera una relación nula (de uno a ninguno), unívoca (de uno a uno) o múltiple (de uno a varios) entre los códigos de las clasificaciones. El algoritmo de funcionamiento del Grouper $\mathrm{ACG}^{\circledR}$ versión 7.1

TABLA I

CARACTERÍSTICAS GENERALES DE LA SERIE Y DESCRIPCIÓN DE ALGUNOS PARÁMETROS DE PLANIFICACIÓN SANITARIOS POR CADA EQUIPO DE ATENCIÓN PRIMARIA ESTUDIADO DURANTE EL AÑO 2006. DERIVACIONES GENERALES Y NEUROLÓGICAS

\begin{tabular}{|c|c|c|c|c|c|c|c|}
\hline Características generales de los EAP & EAP-01 & EAP-02 & EAP-03 & EAP-04 & EAP-05 & Total & p \\
\hline $\begin{array}{l}\text { Número de derivaciones anuales totales } \\
\% \text { sobre el total } \\
\text { Número de derivaciones anuales (especialidad de neurología) } \\
\text { Población asignada (habitantes) } \\
\text { Pacientes atendidos } \\
\text { Visitas realizadas } \\
\text { Episodios atendidos } \\
\text { Problemas de salud/contactos (motivos de consulta) }\end{array}$ & $\begin{array}{c}10.620 \\
18,6 \% \\
212 \\
21.457 \\
15.632 \\
123.299 \\
71.015 \\
145.997\end{array}$ & $\begin{array}{c}6.415 \\
11,3 \% \\
150 \\
13.305 \\
10.633 \\
95.638 \\
54.788 \\
119.445\end{array}$ & $\begin{array}{c}7.924 \\
13,9 \% \\
201 \\
17.060 \\
12.881 \\
100.985 \\
57.857 \\
120.700\end{array}$ & $\begin{array}{c}19.376 \\
34,0 \% \\
584 \\
30.968 \\
22.467 \\
168.121 \\
94.879 \\
195.756\end{array}$ & $\begin{array}{l}12.615 \\
22,2 \% \\
289 \\
28.794 \\
19.162 \\
147.027 \\
87.647 \\
187.636\end{array}$ & $\begin{array}{c}56.950 \\
100,0 \% \\
1.436 \\
111.584 \\
80.775 \\
635.070 \\
366.186 \\
769.534\end{array}$ & \\
\hline $\begin{array}{l}\text { Edad en años, promedio } \pm \text { DT } \\
\text { Cénero (mujeres, \%) } \\
\text { Centro receptor (propia organización, \%) } \\
\text { Prioridad (normal/preferente, \%) } \\
\text { Especialidad de origen/emisora (médicos de familia, \%) }\end{array}$ & $\begin{array}{l}37,8 \pm 21,8 \\
51,3 \\
77,7 \\
76,7 \\
89,7\end{array}$ & $\begin{array}{l}41,5 \pm 23,2 \\
53,2 \\
78,5 \\
82,6 \\
90,4\end{array}$ & $\begin{array}{l}39,8 \pm 22,9 \\
53,3 \\
80,6 \\
73,7 \\
90,3\end{array}$ & $\begin{array}{c}39,6 \pm 22,3 \\
52,8 \\
81,8 \\
78,7 \\
89,7\end{array}$ & $\begin{array}{c}44,3 \pm 23,7 \\
54,7 \\
88,7 \\
75,1 \\
90,9\end{array}$ & $\begin{array}{l}40,7 \pm 22,9 \\
53,1 \\
82,2 \\
77,2 \\
90,3\end{array}$ & $\begin{array}{l}0,000 \\
0,011 \\
0,000 \\
0,000 \\
\text { NS }\end{array}$ \\
\hline $\begin{array}{l}\text { Parámetros de planificación anuales } \\
\text { Intensidad de uso (pacientes atendidos/población asignada, \%) } \\
\text { Frecuentación (ratio entre el número de visitas/población asignada) } \\
\text { Pacientes atendidos con al menos una derivación, (\%) } \\
\text { Número de derivaciones por cada } 100 \text { habitantes asignados } \\
\text { Número de derivaciones por cada } 100 \text { pacientes atendidos } \\
\text { Número de derivaciones por cada } 100 \text { visitas realizadas (TD) }\end{array}$ & $\begin{array}{c}72,9 \% \\
5,7 \\
41,5 \% \\
49,5 \\
67,9 \\
8,6\end{array}$ & $\begin{array}{c}79,9 \% \\
7,2 \\
37,8 \% \\
48,2 \\
60,3 \\
6,7\end{array}$ & $\begin{array}{c}75,5 \% \\
5,9 \\
38,6 \% \\
46,4 \\
61,5 \\
7,8\end{array}$ & $\begin{array}{c}72,5 \% \\
5,4 \\
47,7 \% \\
62,6 \\
86,2 \\
11,5\end{array}$ & $\begin{array}{c}66,5 \% \\
5,1 \\
42,3 \% \\
43,8 \\
65,8 \\
8,6\end{array}$ & $\begin{array}{c}72,4 \% \\
5,7 \\
42,5 \% \\
51,0 \\
70,5 \\
9,0 \\
\end{array}$ & $\begin{array}{l}0,000 \\
0,014 \\
0,003 \\
0,000 \\
0,000 \\
0,000\end{array}$ \\
\hline $\begin{array}{l}\text { Derivaciones neurológicas (derivaciones neurológicas/totales, \%) } \\
\text { Pacientes atendidos con al menos una derivación neurológica, }(\%) \\
\text { Número de derivaciones neurológicas por cada } 100 \text { habitantes asignados } \\
\text { Número de derivaciones neurológicas por cada } 100 \text { pacientes atendidos } \\
\text { Número de derivaciones neurológicas por cada } 100 \text { visitas realizadas }\end{array}$ & $\begin{array}{l}2,0 \% \\
1,3 \% \\
0,99 \\
1,36 \\
0,17\end{array}$ & $\begin{array}{l}2,3 \% \\
1,4 \% \\
1,13 \\
1,41 \\
0,16\end{array}$ & $\begin{array}{l}2,5 \% \\
1,5 \% \\
1,18 \\
1,56 \\
0,20\end{array}$ & $\begin{array}{c}3,0 \% \\
2,4 \% \\
1,89 \\
2,60 \\
0,35\end{array}$ & $\begin{array}{l}2,3 \% \\
1,5 \% \\
1,00 \\
1,51 \\
0,20\end{array}$ & $\begin{array}{l}2,5 \% \\
1,7 \% \\
1,29 \\
1,78 \\
0,23\end{array}$ & $\begin{array}{l}0,015 \\
0,000 \\
0,001 \\
0,017 \\
0,027\end{array}$ \\
\hline
\end{tabular}

EAP: equipos de atención primaria; DT: desviación típica; p: grado de significación estadística; TD: tasa de derivación anual; NS: no significativo 
(http: //www.acg.jhph.edu), está constituido por una serie de pasos consecutivos hasta la obtención de los 106 ACG, grupos mútuamente excluyentes para cada paciente atendido. Para la construcción de un ACG se requiere la edad, el sexo y los motivos de consulta o diagnósticos codificados según la CIE-9-MC. El proceso de clasificación de CIE-9-MC en ACG consta de 4 etapas, las dos primeras con el propósito de agrupar una serie de condiciones por similar consumo de recursos y las dos últimas con el objetivo de combinar las agrupaciones más frecuentes, finalmente cada paciente es asignado a un solo grupo de isoconsumo de recursos (Fig. 2).

El proceso de CIE-9-MC en ACGs consta de 4 etapas, las dos primeras
con el propósito de agrupar una serie de condiciones por similar consumo
de recursos y las dos últimas con el objetivo de combinar las agrupaciones
más frecuentes

CIAP: Clasificación Internacional de la Atención Primaria CIE-9-MC: Clasificación Internacional de Enfermedades, novena edición, modificación clínica

Fig. 2. Breve algoritmo de funcionamiento del Grouper ACGs (versión 7.1)

El promedio de derivaciones (número de derivaciones/pacientes atendidos) relativo de cada ACG se obtuvo dividiendo el promedio de derivaciones de cada categoría entre el promedio de derivaciones de toda la población de referencia (conjunto de los cinco centros). Por tanto, el índice de eficiencia (IE) se estableció como el cociente entre las derivaciones observadas y las esperadas en función de la distribución ACG para el conjunto de pacientes (estandarización indirecta). Un valor de IE igual a 1 significa igual eficiencia que la norma o estándar (conjunto de los centros), mientras que un IE inferior a 1 simboliza una mayor eficiencia (relación inversa).

Como paso previo al análisis, y en particular a la fuente de información perteneciente a las historias clínicas informatizadas, se revisaron cuidadosamente los datos, observando sus distribuciones de frecuencia y buscando posibles errores de registro o de codificación. Los datos se obtuvieron de forma informatizada, respetándose la confidencialidad de los regis- tros marcada por la ley. Las variables estudiadas han sido exploradas con la prueba de conformidad de KolmogorovSmirnov. El cálculo del poder explicativo de la clasificación se ha establecido a través del cociente de determinación entre la varianza intragrupo y la varianza total, la transformación de las variables se realizó a partir del logaritmo neperiano y se detallaron los intervalos de confianza del 95\% (IC). La relación de variables cuantitativas se efectuó con la correlación ordinal no paramétrica de Spearman y el análisis de ANOVA, y las variables cualitativas con la prueba de ji-cuadrado. Además, se efectuó un análisis de regresión lineal múltiple (procedimiento enter) para la predicción de los cuatro modelos utilizados: modelo 1, considera como variable independiente la edad; modelo 2: la edad y el género; modelo 3: la edad, el género y el número de ADGs; y el modelo 4: la edad, el género, el número de ADGs y de episodios de atención. La agrupación y relación de las medidas se efectuó con el programa informático Microsoft Access ${ }^{\circledR}$. El análisis estadístico se realizó con el programa SPSSW versión 12, aceptándose un nivel de significación del $5 \%$.

\section{RESULTADOS}

El número de habitantes adscritos a los EAP durante el año 2006 fue de 110.440 (medicina de fanilia: 84,7\%; pediatría: $15,3 \%$ ). Durante el período de estudio, se contabilizaron 387.720 problemas de salud y 638.123 visitas, en 80.775 pacientes atendidos. En la tabla I se detallan las características generales de la serie estudiada, así como algunos indicadores unitarios, observandose una cierta variabilidad en las características de los EAP. La frecuentación fue de 5,7 visitas/habitante/año, siendo superior en el servicio de pediatría 9,2 visitas/habitante/año. La intensidad de uso de servicios sanitarios en los cinco centros fue del 72,4\% (IC: $72,1-72,7 \%$ ), con una media de 4,8 $\pm 3,5$ episodios y 7,9 $\pm 8,2$ visitas realizadas por cada paciente atendido/año $(\mathrm{n}=80.775), \mathrm{p}=0,000$. El promedio de edad de la serie fue de $40,7 \pm 22,9$ años y un $53,1 \%$ fueron mujeres. La TD general fue del 9,0\% (IC: 8,8-9,2; rango: $6,7-11,5 \% ; \mathrm{n}=56.950$ derivaciones). La edad media de los pacientes con derivación fue de 44,8 \pm 22,8 años, un $54,6 \%$ mujeres $(\mathrm{p}=0,000)$. El promedio de derivaciones $/ 100$ pacientes atendidos/año fue de 70,5 ( $\mathrm{p}=0,000)$. Un $2,5 \%$ sobre el total de derivaciones se realizaron a la especialidad de neurologia (IC: 1,7-3,3; rango: 2,0-3,0; $\mathrm{n}=1.436$ derivaciones). La TD neurológica fue del $0,23 \%$ (IC: $0,0-0,5 \%$ ), p = 0,027 . La edad media de los pacientes con derivación neurológica fue de 48,2 $\pm 23,6$ años, un 55,9\% mujeres $(p=0,001)$. El promedio de derivaciones neurológicas por cada 100 pacientes atendidos/año fue de 1,78 $(\mathrm{p}=0,017)$.

La distribución de las derivaciones por cada servicio emisor se muestra en la tabla II. Del total de derivaciones efectuadas a la atención especializada $(n=56.950)$, el 89,3\% se realizaron por médicos de familia y el $10,7 \%$ por pediatras. Las derivaciones en pacientes adultos predominan en el sexo femenino $(57,0 \%)$, en especialidades de la propia organización $(82,2 \%)$ y son mayoritariamente de tipo quirúrgico. La tres especialidades más demandadas fueron cirugía ortopédica y traumatologia, oftalmología y dermatología, pero en diferente orden según los servicios de medicina de familia o pediatría (correlación ordinal de Spearman, $\mathrm{p}=0$,002) (Tabla II). Los principales motivos de derivación realizados a la 
TABLA II

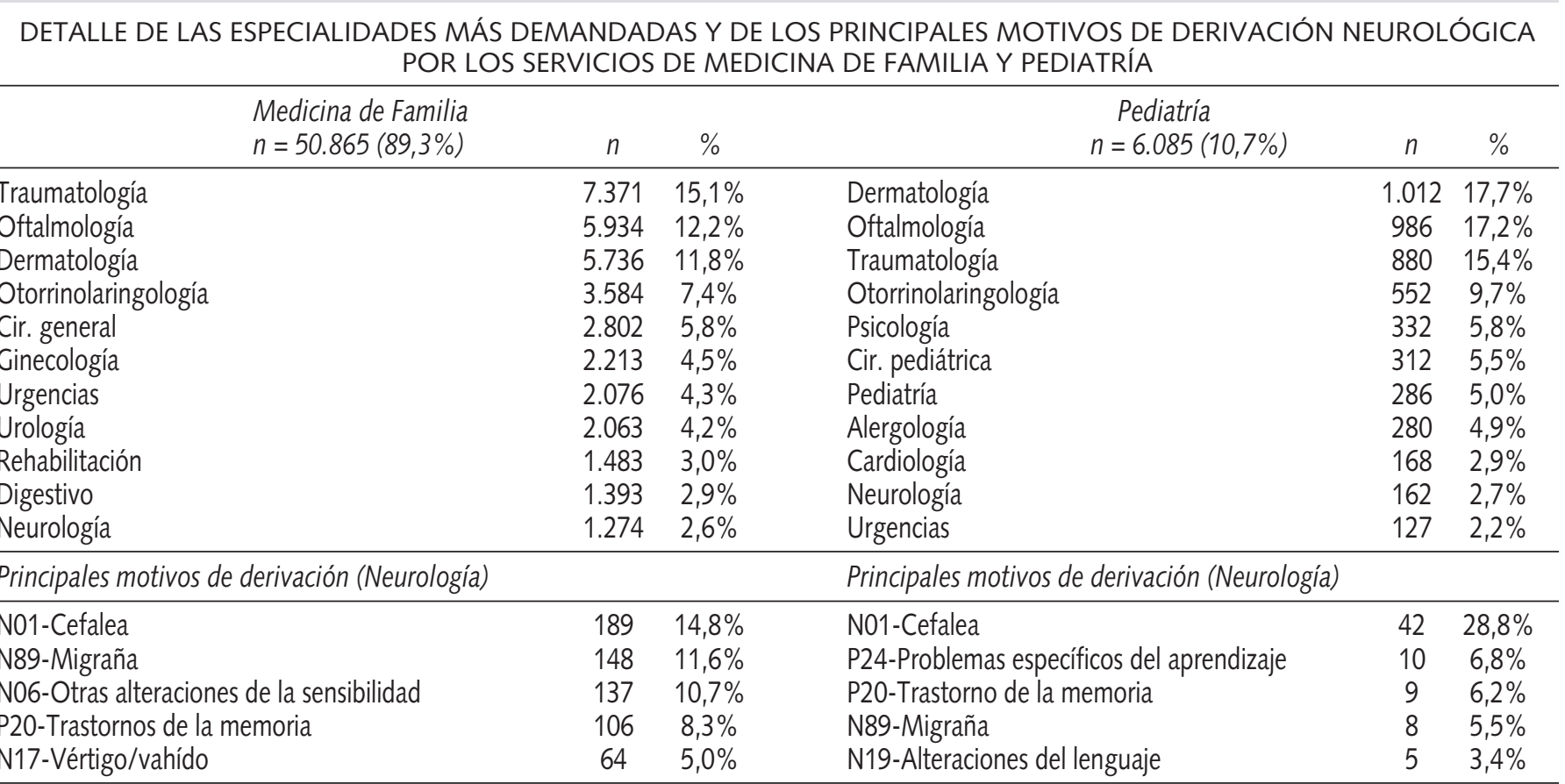

$\mathrm{n}=56.950$ derivaciones a especialistas de referencia. $\mathrm{n}=1.436(2,5 \%)$ derivaciones a neurología. $\mathrm{n}$ : número de derivaciones; motivos de derivación según la CIAP (Clasificación Internacional de la Atención Primaria).

\begin{tabular}{|c|c|c|c|}
\hline \multicolumn{4}{|c|}{ TABLA III } \\
\hline \multicolumn{4}{|c|}{$\begin{array}{l}\text { CÁLCULO DEL COEFICIENTE DE DETERMINACIÓN PARA } \\
\text { CADA VARIABLE DEPENDIENTE POR LOS SERVICIOS DE } \\
\text { MEDICINA DE FAMILIA Y PEDIATRÍA. MEDIDA DEL PODER } \\
\text { EXPLICATIVO DE LA CLASIFICACIÓN (REGRESIÓN LINEAL) } \\
\text { SEGÚN LOS CUATRO MODELOS DE ESTUDIO DE LA } \\
\text { CLASIFICACIÓN ACGS (AJUSTED CLINICAL GROUPS) }\end{array}$} \\
\hline Variables dependientes & $\begin{array}{c}\text { Medicina } \\
n=67.854\end{array}$ & $\begin{array}{c}\text { Pediatría } \\
n=12.921\end{array}$ & $\begin{array}{c}\text { Total } \\
n=80.775\end{array}$ \\
\hline Promedio de visitas & $43,7 \%$ & $41,3 \%$ & $43,2 \%$ \\
\hline Promedio de episodios & $79,0 \%$ & $52,0 \%$ & $73,9 \%$ \\
\hline Promedio de derivaciones & $19,7 \%$ & $16,1 \%$ & $20,1 \%$ \\
\hline \multicolumn{4}{|l|}{ Trasformación de variables } \\
\hline Promedio de visitas & $58,5 \%$ & $45,7 \%$ & $55,9 \%$ \\
\hline Promedio de episodios & $80,3 \%$ & $60,4 \%$ & $76,5 \%$ \\
\hline Promedio de derivaciones & $12,8 \%$ & $7,9 \%$ & $12,7 \%$ \\
\hline \multicolumn{4}{|c|}{ Varianza explicada de la clasificación (regresión lineal) } \\
\hline Modelo 1: edad & & & $9,0 \%$ \\
\hline Modelo 2: edad y género & & & $13,1 \%$ \\
\hline Modelo 3: edad, género y & ADGs & & $42,0 \%$ \\
\hline Modelo 4: edad, género, n & DGs y episodi & & $46,3 \%$ \\
\hline \multicolumn{4}{|c|}{$\begin{array}{l}\text { Coeficiente de determinación de las variables dependientes (R2) obtenido } \\
\text { a partir de la varianza explicada/varianza total (prueba de Ficher-Snede- } \\
\text { cor); significación estadística, } p<0,001 \text {. Transformación de variables } \\
\text { dependientes a partir de logaritmo neperiano. Regresión lineal múltiple: } \\
\text { método de entrada de las variables: enter (estimación: mínimos cuadrados; } \\
\text { índice de condicionamiento: inferior a 10). ADGs: Ambulatory Diagnostic } \\
\text { Groups; significación estadística en todos los casos: } p<0,001 \text {. }\end{array}$} \\
\hline
\end{tabular}

especialidad de neurología desde la atención primaria al segundo nivel por los médicos de familia o pediatras se detalla en la tabla II. Destacan las efectuadas por los pacientes con cefalea/migraña $(n=1.436 ; 26,4 \%)$.

Para medir el ajuste o poder explicativo de la clasificación se confeccionaron dos modelos (Tabla III), uno en relación a las variables dependientes, donde las visitas y los episodios explican el 43,2 y el 73,9\% respectivamente $(\mathrm{p}=0,000)$, y otro para medir el poder explicativo de la variabilidad de la clasificación ACG que fue del 46,3\% al incluir los episodios en el modelo 4 ( $\mathrm{p}<0,0001)$. Cabe destacar que el ajuste de las derivaciones explica el $20,1 \%$ de la variabilidad. Finalmente, en la figura 3 se describen los IE (cociente entre las derivaciones observadas y las esperadas para el conjunto de pacientes atendidos). Una vez ajustadas las derivaciones por edad, sexo y comorbilidades (casuística) durante el año de estudio, se observan unos IE por médicos de familia del 0,97 (IC: 0,77-1,18), 0,79 (IC: 0,571,01), 0,88 (IC: 0,62-1,14), 1,29 (IC: 0,94-1,65) y 0,91 (IC: $0,58-1,25), p=0,023$; y para pediatría del 0,90 (IC: $0,47-1,33$ ), 0,78 (IC: 0,35-1,21), 0,93 (IC: 0,43-1,44), 1,21 (IC: 0,60-1,82) y 0,97 (IC: 0,39-1,56), p = 0,031; en los respectivos centros. En general, los EAP-2 y EAP-3 (IE = 0,79 y 0,88) se muestran más eficientes en las derivaciones efectuadas a los especialistas de referencia con respecto a la norma, los EAP-1 y EAP-5 (IE = $0,96$ y 0,92$)$ moderadamente eficientes y el EAP-4 $(\mathrm{IE}=1,28)$ claramente ineficiente. La distribución final de los pacientes según la Clasificación ACG se detalla en el Anexo I.

\section{DISCUSIÓN}

Conceptualmente los médicos de familia en su práctica clínica efectúan a priori una selección de pacientes, de manera que una derivación excesiva puede producir un uso de recursos y un 
riesgo innecesario, mientras que una demora puede retrasar un diagnóstico y/o tratamiento acertado, y acabar ocasionando intervenciones menos eficientes $(12,13,23-25)$. En este aspecto, en Estados Unidos, la Agency for Health Care Policy and Research (AHCPR, 1996), convocó ayudas para la investigación en demanda derivada y fruto de esa iniciativa surgieron diversos estudios a gran escala. En general, se orientaron a describir las tasas y el patrón de derivación, y la mayoría evidenciaron una gran variabilidad interprofesional y una mayor derivación en las especialidades quirúrgicas, resultados coincidentes con la mayoría de los estudios publicados (8-17,26-28). El modelo organizativo en el que se encuentran la mayoría de los EAP de nuestro país, en base a la asignación poblacional en base territorial y a la creciente informatización de sus centros, ofrece un marco idó-

Índice de eficiencia (IE): Distribución General

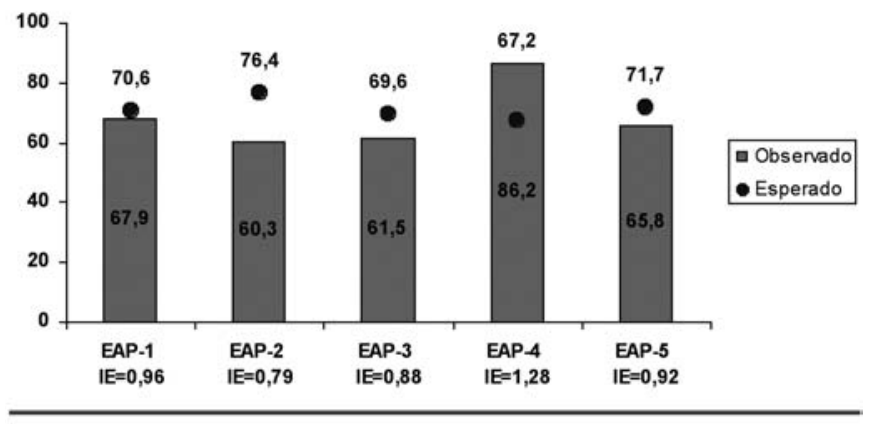

Índice de eficiencia (IE): Medicina de Familia

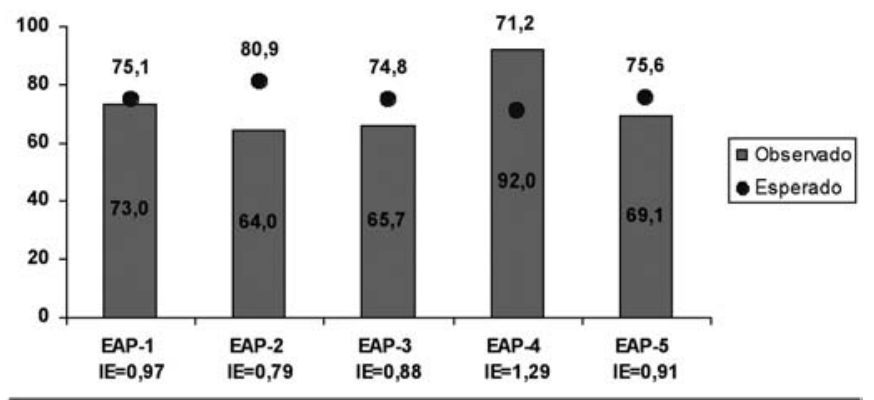

Índice de eficiencia (IE): Pediatría

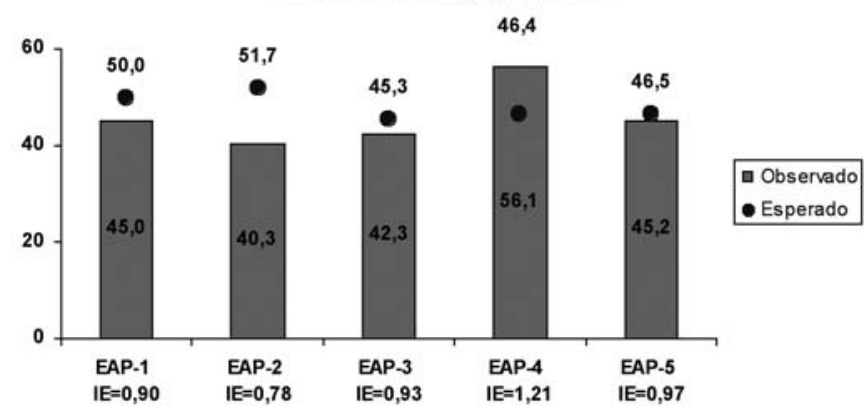

Fig. 3. Índices de eficiencia (IE) de las derivaciones a especialistas de referencia en los diferentes centros por Médicos de Familia y Pediatría

EAP: Equipos de Atención Primaria; IE: cociente entre las derivaciones/paciente observadas y las esperadas en función de la distribución ACGs (casuística ajustada por edad, sexo y comorbilidad) para el conjunto de pacientes atendidos (estandarización indirecta). Valores de IE igual a 1 significa igual eficiencia que la norma o estándar (conjunto de los centros), un IE inferior a 1 simboliza una mayor eficiencia (relación inversa). neo para poder realizar estudios retrospectivos en situación de práctica clínica habitual (29). Los estudios existentes en España de aplicación práctica (eficiencia) sobre ACG son escasos; además sin una adecuada estandarización de metodologías (30), en cuanto a las características de los centros y medida de las variables utilizadas, los resultados deben de interpretarse con prudencia y dentro del escenario de política sanitaria, proveedor de servicios, cultura de la organización y de gestión clínica que le son propios, limitando la generalización externa de los resultados.

En la revisión bibliográfica efectuada, llama la atención la variabilidad existente en la TD efectuadas a los especialistas de referencia. Aspecto que ha podido constatarse en los datos brutos de nuestro estudio (TD: 9,0\%; rango: $6,7-11,5 \% ; \mathrm{n}=$ 56.950 ; promedio de derivaciones por cada 100 pacientes atendidos/año del 70,5; observándose diferencias entre los EAP, $\mathrm{p}<$ $0,001)$, posiblemente atribuibles a discrepancias de práctica clínica y/o de accesibilidad. Diversos estudios realizados en Reino Unido y en Estados Unidos demuestran que a igualdad de morbilidad de la población, los médicos de familia ingleses derivan un $16 \%$ menos que los norteamericanos $(16,25,26)$. Parece evidente que a mayor carga de morbilidad, mayor utilización de los recursos, mayor número de visitas y mayor necesidad de derivación a la atención especializada. Este aspecto también ha sido observado en el estudio, ya que predomina una fuerte correlación entre las visitas realizadas con los episodios/motivos de consulta atendidos. Ante este escenario, es probable que el entorno cultural, las expectativas de los usuarios, la formación de los profesionales u otros aspectos influyan, pero pueden resultar fundamentales los mecanismos de coordinación entre los diferentes niveles asistenciales (8-11,13-16). Mientras que en Inglaterra la atención primaria compra los servicios de la especializada, las estadounidenses se organizan en sistemas que unifican la atención primaria y la especializada; los británicos dan un mayor poder a los médicos de familia, mientras que los americanos a la atención especializada $(16,25)$; en nuestro medio las innovaciones en gestión van más orientadas al modelo británico.

Es importante destacar que únicamente un 2,5\% del total de derivaciones se realizaron a la especialidad de neurología (rango: $2,0-3,0 ; n=1.436$ derivaciones/año), la TD fue del $0,23 \% \mathrm{y}$ el promedio de derivaciones por cada 100 pacientes atendidos/año de 1,78; siendo pacientes de mayor edad, con predominio de mujeres y presentando la cefalea/migraña como principal motivo de consulta. Nuestra serie se sitúa en unos niveles de TD bajos, en cambio los demás aspectos comentados son coincidentes con diversos autores (23-26). Esta baja TD podría deberse principalmente a un posible aumento de la capacidad resolutiva de los profesionales o a aspectos organizativos; en la organización se disponen de unidades específicas multidisciplinares de geriatría, demencia, atención domiciliaria, y se tiene acceso a determinadas pruebas complementarias (electromiografía), con lo que el servicio de neurología de la atención especializada puede recibir pacientes con un mayor grado de severidad o más específicos para estudio.

El poder explicativo de la clasificación en relación a las variables dependientes, visitas y episodios, explican el 43,2 y el $73,9 \%$ respectivamente, además, la variabilidad de la clasificación ACG fue del 46,3\% al incluir los episodios en el modelo 4 $(\mathrm{p}<0,0001)$. Estos aspectos, reafirman la importancia de cuantificar los diagnósticos; la edad y el género, no son suficientes para cuantíficar el uso de los recursos sanitarios. Estos datos son consistentes con diversas evidencias disponibles. Por tanto, 
estandarizar la forma de presentar los perfiles de derivación entre EAP puede proporcionar resultados más aceptables y comparables, ya que sería arriesgado concluir que un centro que tenga una tasa de derivación menor, es más eficiente y resolutivo. En el estudio, una vez ajustadas las derivaciones por edad, sexo y comorbilidades (casuística), se observan unos IE por médicos de familia y pediatras en los respectivos centros; donde los EAP 2 y 3 se muestran más eficientes. Conceptualmente los ACG tienen una gran validez clínica ya que los patrones de morbilidad son relativamente constantes en el tiempo y las personas incluidas en un grupo tienen una gran probabilidad de permanecer en dicho grupo a lo largo de los años siguientes (8$11,14,17,27,28,31,32)$. Por tanto, este tipo de estudios deben valorarse en un contexto positivo. Un aspecto de interés práctico consiste en que el agrupador necesita un número de variables limitado para cada paciente: edad, sexo y diagnósticos. Esta simplicidad de uso se ajusta a las necesidades de la atención primaria de salud, con almacenamiento de un gran volumen de información, limitación en el tiempo de visita asistencial, coexistencia de profesionales en un mismo proceso clínico y reiteración de pacientes a lo largo del tiempo $(3-5,33,34)$.

Las limitaciones más destacadas deben de relacionarse con el grado de maduración de los sistemas de información desarrollados en el estudio, la posible variabilidad y/o gravedad en la selección del episodio de atención por parte de los diferentes facultativos, que puede ocasionar efectos de contaminación entre los grupos o la existencia de poca especificidad clínica (30). Pero la mayor limitación se presenta en la validez externa de los resultados, en dos aspectos; por una parte, los centros estudiados no son representativos de un universo general, son centros pertenecientes a una organización sanitaria particular, y por otra, el número de pacientes incluidos en el estudio es poco elevado para este tipo de generalizaciones. Futuras investigaciones deberían de evaluar en detalle los mecanismos de coordinación de los diferentes niveles asistenciales para tratar la comorbilidad asociada a los pacientes y disponer de un mayor grado de desarrollo y aplicabilidad de los sistemas de información y de clasificación de pacientes por isoconsumo de recursos para un mejor ajuste del modelo, por tanto, el estudio de la eficiencia permite profundizar en el conocimiento del perfil de los profesionales y de los centros de salud (35). En conclusión, los ACG se muestran como un aceptable sistema de clasificación de pacientes en situación de práctica clínica habitual. La tasa y el patrón de derivación general fue similar al descrito en estudios anteriores, aunque solo un $2,5 \%$ del total de derivaciones fueron realizadas a la especialidad de neurología, la TD fue del 0,23\% y el promedio de derivaciones por cada 100 pacientes atendidos/año de 1,78; siendo pacientes de mayor edad, con predominio de mujeres y presentando la cefalea/migraña como principal motivo de consulta. La morbilidad explica el 73,9\% de la variación total y el poder explicativo de la clasificación ACG una parte importante de las derivaciones. Los resultados del estudio deben de interpretarse con prudencia incluso después del ajuste por edad, sexo y morbilidad. De confirmarse los resultados posibilitarían una mejora en la medida de las derivaciones para la gestión clínica de los centros de atención primaria.

\section{AGRADECIMIENTOS}

A los diferentes profesionales sanitarios de nuestra organización, ya que sin su constante introducción de los datos en el día a día, no hubiera sido posible la realización de este estudio.

\section{ANEXO I}

DISTRIBUCIÓN DE LOS PACIENTES SEGÚN LA CLASIFICACIÓN ACG. PROMEDIO DE VISITAS Y EPISODIOS (VARIABLES DEPENDIENTES) POR CADA GRUPO

\begin{tabular}{ll}
\hline ACG & General description \\
0100 & Acute Minor, Age 1 \\
0200 & Acute Minor, Age 2 to 5 \\
0300 & Acute Minor, Age 6+ \\
0400 & Acute Major \\
0500 & Likely to Recur, w/o Allergies \\
0600 & Likely to Recur, w/ Allergies \\
0700 & Asthma \\
0800 & Chronic Medical: Unstable \\
0900 & Chronic Medical: Stable \\
1000 & Chronic Specialty: Stable \\
1100 & Eye \& Dental \\
1200 & Chronic Specialty: Unstable \\
1300 & Psychosocial, w/o Psychosocial Unstable \\
1400 & Psychosocial, w/ Psychosocial Unstable, w/o Psychosocial Stable \\
1500 & Psychosocial, w/ Psychosocial Unstable, w/ Psychosocial Stable \\
1600 & Preventive/Administrative \\
1710 & Pregnancy, 0-1 ADGs \\
1720 & Pregnancy, 2-3 ADGs, no Major ADGs
\end{tabular}

$\begin{array}{rrrr}\mathrm{N} & \% & \mathrm{Vx} & \mathrm{Dx} \\ 259 & 0,3 \% & 13,4 & 4,4 \\ 878 & 1,1 \% & 6,1 & 3,5 \\ 10.152 & 12,6 \% & 2,6 & 1,8 \\ 2.575 & 3,2 \% & 2,6 & 1,6 \\ 3.507 & 4,3 \% & 2,3 & 1,5 \\ 413 & 0,5 \% & 3,4 & 1,8 \\ 139 & 0,2 \% & 2,7 & 1,4 \\ 461 & 0,6 \% & 3,1 & 1,5 \\ 1.854 & 2,3 \% & 3,9 & 2,0 \\ 110 & 0,1 \% & 2,7 & 1,3 \\ 104 & 0,1 \% & 2,3 & 1,2 \\ 237 & 0,3 \% & 3,1 & 1,4 \\ 1.003 & 1,2 \% & 2,8 & 1,5 \\ 85 & 0,1 \% & 3,9 & 1,6 \\ 8 & 0,0 \% & 5,0 & 3,4 \\ 2.299 & 2,8 \% & 1,7 & 1,0 \\ 165 & 0,2 \% & 2,2 & 1,4 \\ 461 & 0,6 \% & 5,2 & 3,3\end{array}$




\begin{tabular}{|c|c|c|c|c|c|}
\hline \multicolumn{6}{|c|}{ ANEXO I (CONTINUACIÓN) } \\
\hline 1730 & Pregnancy, 2-3 ADGs, 1+ Major ADGs & 42 & $0,1 \%$ & 4,8 & 3,4 \\
\hline 1740 & Pregnancy, 4-5 ADGs, no Major ADGs & 266 & $0,3 \%$ & 8,6 & 6,0 \\
\hline 1750 & Pregnancy, 4-5 ADGs, 1+ Major ADGs & 51 & $0,1 \%$ & 8,8 & 5,7 \\
\hline 1760 & Pregnancy, 6+ ADGs, no Major ADGs & 103 & $0,1 \%$ & 12,6 & 9,3 \\
\hline 1770 & Pregnancy, 6+ ADGs, 1+ Major ADGs & 51 & $0,1 \%$ & 15,6 & 10,6 \\
\hline 1800 & Acute Minor/Acute Major & 3.564 & $4,4 \%$ & 5,8 & 3,9 \\
\hline 1900 & Acute Minor/Likely to Recur, Age 1 & 371 & $0,5 \%$ & 22,7 & 8,4 \\
\hline 2000 & Acute Minor/Likely to Recur, Age 2 to 5 & 1.215 & $1,5 \%$ & 11,7 & 7,2 \\
\hline 2100 & Acute Minor/Likely to Recur, Age 6+, w/o Allergy & 4.795 & $5,9 \%$ & 5,0 & 3,7 \\
\hline 2200 & Acute Minor/Likely to Recur, Age 6+, w/ Allergy & 621 & $0,8 \%$ & 6,4 & 4,3 \\
\hline 2300 & Acute Minor/Chronic Medical: Stable & 1.988 & $2,5 \%$ & 6,5 & 3,8 \\
\hline 2400 & Acute Minor/Eye \& Dental & 116 & $0,1 \%$ & 5,5 & 3,6 \\
\hline 2500 & Acute Minor/Psychosocial, w/o Psychosocial Unstable & 1.229 & $1,5 \%$ & 5,6 & 3,5 \\
\hline 2600 & Acute Minor/Psychosocial, w/ Psychosocial Unstable, w/o Psychosocial Stable & 76 & $0,1 \%$ & 6,6 & 3,3 \\
\hline 2700 & Acute Minor/Psychosocial, w/ Psychosocial Unstable/Psychosocial Stable & 24 & $0,0 \%$ & 7,3 & 4,8 \\
\hline 2800 & Acute Major/Likely to Recur & 1.276 & $1,6 \%$ & 4,5 & 3,1 \\
\hline 2900 & Acute Minor/Acute Major/Likely to Recur, Age 1 & 116 & $0,1 \%$ & 27,2 & 10,7 \\
\hline 3000 & Acute Minor/Acute Major/Likely to Recur, Age 2 to 5 & 488 & $0,6 \%$ & 14,9 & 9,4 \\
\hline 3100 & Acute Minor/Acute Major/Likely to Recur, Age 6 to 11 & 528 & $0,7 \%$ & 9,6 & 6,9 \\
\hline 3200 & Acute Minor/Acute Major/Likely to Recur, Age 12+, w/o Allergy & 2.150 & $2,7 \%$ & 7,5 & 5,5 \\
\hline 3300 & Acute Minor/Acute Major/Likely to Recur, Age 12+, w/ Allergy & 304 & $0,4 \%$ & 8,4 & 5,9 \\
\hline 3400 & Acute Minor/Likely to Recur/Eye \& Dental & 122 & $0,2 \%$ & 9,0 & 6,0 \\
\hline 3500 & Acute Minor/Likely to Recur/Psychosocial & 1.272 & $1,6 \%$ & 8,6 & 5,7 \\
\hline 3600 & Acute Minor/Acute Major/Likely to Recur/Chronic Medical: Stable & 2.273 & $2,8 \%$ & 12,4 & 8,2 \\
\hline 3700 & Acute Minor/Acute Major/Likely to Recur/Psychosocial & 1.161 & $1,4 \%$ & 11,8 & 8,0 \\
\hline 3800 & 2-3 Other ADG Combinations, Age 1 to 17 & 690 & $0,9 \%$ & 7,3 & 4,1 \\
\hline 3900 & 2-3 Other ADG Combinations, Males Age 18 to 34 & 918 & $1,1 \%$ & 5,3 & 3,3 \\
\hline 4000 & 2-3 Other ADG Combinations, Females Age 18 to 34 & 1.028 & $1,3 \%$ & 4,9 & 3,4 \\
\hline 4100 & 2-3 Other ADG Combinations, Age 35+ & 8.860 & $11,0 \%$ & 6,9 & 4,0 \\
\hline 4210 & 4-5 Other ADG Combinations, Age 1 to 17, no Major ADGs & 619 & $0,8 \%$ & 13,3 & 7,6 \\
\hline 4220 & 4-5 Other ADG Combinations, Age 1 to 17, 1+ Major ADGs & 162 & $0,2 \%$ & 12,4 & 7,4 \\
\hline 4310 & 4-5 Other ADG Combinations, Age 18 to 44 , no Major ADGs & 1.434 & $1,8 \%$ & 8,4 & 6,0 \\
\hline 4320 & 4-5 Other ADG Combinations, Age 18 to 44,1 Major ADGs & 847 & $1,0 \%$ & 9,3 & 5,8 \\
\hline 4330 & 4-5 Other ADG Combinations, Age 18 to 44, 2+ Major ADGs & 114 & $0,1 \%$ & 10,2 & 5,9 \\
\hline 4410 & 4-5 Other ADG Combinations, Age 45+, no Major ADGs & 3.695 & $4,6 \%$ & 10,7 & 6,7 \\
\hline 4420 & 4-5 Other ADG Combinations, Age 45+, 1 Major ADGs & 3.305 & $4,1 \%$ & 12,3 & 6,8 \\
\hline 4430 & 4-5 Other ADG Combinations, Age 45+, 2+ Major ADGs & 695 & $0,9 \%$ & 14,1 & 6,9 \\
\hline 4510 & 6-9 Other ADG Combinations, Age 1 to 5, no Major ADGs & 96 & $0,1 \%$ & 27,4 & 13,3 \\
\hline 4520 & 6-9 Other ADG Combinations, Age 1 to 5, $1+$ Major ADGs & 42 & $0,1 \%$ & 22,9 & 13,0 \\
\hline 4610 & 6-9 Other ADG Combinations, Age 6 to 17, no Major ADGs & 131 & $0,2 \%$ & 15,7 & 10,2 \\
\hline 4620 & 6-9 Other ADG Combinations, Age 6 to 17, 1+ Major ADGs & 58 & $0,1 \%$ & 16,3 & 10,1 \\
\hline 4710 & 6-9 Other ADG Combinations, Males, Age 18 to 34, no Major ADGs & 69 & $0,1 \%$ & 12,0 & 8,8 \\
\hline 4720 & 6-9 Other ADG Combinations, Males, Age 18 to 34, 1 Major ADGs & 56 & $0,1 \%$ & 13,8 & 8,9 \\
\hline 4730 & 6-9 Other ADG Combinations, Males, Age 18 to $34,2+$ Major ADGs & 16 & $0,0 \%$ & 15,9 & 8,6 \\
\hline 4810 & 6-9 Other ADG Combinations, Females, Age 18 to 34, no Major ADGs & 187 & $0,2 \%$ & 13,3 & 9,4 \\
\hline 4820 & 6-9 Other ADG Combinations, Females, Age 18 to 34, 1 Major ADGs & 119 & $0,1 \%$ & 15,4 & 9,8 \\
\hline 4830 & 6-9 Other ADG Combinations, Females, Age 18 to 34, 2+ Major ADGs & 25 & $0,0 \%$ & 14,8 & 10,4 \\
\hline 4910 & 6-9 Other ADG Combinations, Age 35+, 0-1 Major ADGs & 5.501 & $6,8 \%$ & 16,9 & 10,5 \\
\hline 4920 & 6-9 Other ADG Combinations, Age 35+, 2 Major ADGs & 1.320 & $1,6 \%$ & 21,0 & 10,9 \\
\hline 4930 & 6-9 Other ADG Combinations, Age 35+, 3 Major ADGs & 212 & $0,3 \%$ & 23,1 & 11,3 \\
\hline 4940 & 6-9 Other ADG Combinations, Age 35+, 4+ Major ADGs & 21 & $0,0 \%$ & 24,7 & 11,4 \\
\hline 5010 & 10+ Other ADG Combinations, Age 1 to 17, no Major ADGs & 2 & $0,0 \%$ & 41,5 & 22,0 \\
\hline 5020 & 10+ Other ADG Combinations, Age 1 to 17, 1 Major ADGs & 3 & $0,0 \%$ & 50,0 & 18,3 \\
\hline 5040 & 10+ Other ADG Combinations, Age 18+, 0-1 Major ADGs & 349 & $0,4 \%$ & 27,4 & 17,2 \\
\hline 5050 & $10+$ Other ADG Combinations, Age 18+, 2 Major ADGs & 236 & $0,3 \%$ & 30,2 & 17,3 \\
\hline 5060 & 10+ Other ADG Combinations, Age 18+, 3 Major ADGs & 85 & $0,1 \%$ & 38,2 & 18,1 \\
\hline 5070 & $10+$ Other ADG Combinations, Age 18+, 4+ Major ADGs & 15 & $0,0 \%$ & 40,5 & 18,0 \\
\hline 5100 & No Diagnosis or Only Unclassified Diagnosis or Non-Users (1 input file) & 3 & $0,0 \%$ & 1,0 & 1,0 \\
\hline 5310 & Infants: 0-5 ADGs, no Major ADGs & 900 & $1,1 \%$ & 15,2 & 4,0 \\
\hline 5320 & Infants: 0-5 ADGs, 1+ Major ADGs & 24 & $0,0 \%$ & 19,8 & 5,9 \\
\hline 5330 & Infants: 6+ ADGs, no Major ADGs & 24 & $0,0 \%$ & 26,4 & 10,3 \\
\hline \multirow[t]{2}{*}{5340} & Infants: 6+ ADGs, 1+ Major ADGs & 2 & $0,0 \%$ & 17,5 & 11,5 \\
\hline & Total & 80.775 & $100,0 \%$ & 7,9 & 4,8 \\
\hline
\end{tabular}

N: número de pacientes agrupados; \%: porcentaje de los casos; ACG: Adjusted Clinical Groups; ADGs: Ambulatory Diagnostic Groups; Vx: promedio de visitas; Dx: promedio de episodios. Se eliminaron de la clasificación los grupos ACG que no mostraron pacientes. 


\section{Bibliografía}

1. Meenan RT, Goodman MJ, Fishman PA, Hornbrook MC, O'KeeffeRosetti MC, Bachman DJ. Using risk-adjustment models to identify high-cost risks. Med Care 2003; 41: 1301-12.

2. Petersen LA, Pietz K, Woodard LD, Byrne M. Comparison of the predictive validity of diagnosis-based risk adjusters for clinical outcomes. Med Care 2005; 43: 61-7

3. Sicras-Mainar A, Serrat-Tarres J, Navarro-Artieda R, Llopart-Lopez J. Prospects of adjusted clinical groups (ACG's) in capitated payment risk adjustment. Rev Esp Salud Publica 2006; 80: 55-65.

4. Starfield B, Weiner J, Mumford L, Steinwachs D. Ambulatory Care Groups: a categorization of diagnoses for research and management. Health Ser Res 1991; 26: 53-74.

5. Weiner JP, Starfield BH, Steinwachs DM, Mumford LM. Development and application of a population-oriented measure of ambulatory care case-mix. Med Care 1991; 29: 452-72.

6. Hughes JS, Averill RF, Eisenhanler J, Goldfield NI, Muldoon J, Neft JM, Gay JC. Clinical Risk Groups (CRGs): a classification system for risk adjusted capitation-based payment and health care management. Med Care 2004; 42: 81-90

7. Wahls TL, Barnett MJ, Rosenthal GE. Predicting resource utilization in a veterans health administration primary care population: comparison of methods based on diagnoses and medications. Med Care 2004; 42: 123-8.

8. Sánchez-Sánchez C. Impacto sociosanitario de las enfermedades neurológicas en España. URL: http: //www.feeneurologia.com/pdf/impactsociosanitarioenferneurologicas.pdf. Consultado el 6.7.2007.

9. Pérez-Martínez DA, Puente-Muñoz AI. La autorización previa para acceder al neurólogo desde atención primaria puede ser innecesaria: resultados de un estudio prospectivo y controlado. Rev Neurol 2006; 43: 388-92.

10. Morera-Guitart J. Asistencia neurológica ambulatoria en España. Pasado, presente, ¿futuro? Rev Neurol 2005; 41: 65-7.

11. De Prado-Prieto L, García-Olmos L, Rodríguez- Salvanés F, OteroPuime A. Evaluación de la demanda derivada en atención primaria. Aten Primaria 2005; 35: 146-51.

12. Lin CT, Albertson G, Price D, Swaney R, Anderson S, Anderson RJ. Patient desire and reasons for specialist referral in a gatekeeper-model managed care plan. Am J Manag Care 2000; 6: 669-78.

13. Alonso JP, Febrel M, Domeco JH. Factores asociados a la derivación inadecuada entre atención primaria y especializada: estudio cualitativo en médicos de atención primaria. Gac Sanit 2000; 14: 122-30

14. Ferradal García JL, Peral Casado A, Villa Martínez B, Burón Llamazares JL. Variabilidad en la derivación de los profesionales de un centro de salud rural. SEMERGEN 2005; 31: 351-55

15. Bolanos-Carmona V, Ocaña-Riola R, Prados-Torres A, Gutiérrez-Cuadra P. Variations in health services utilization by primary care patients. Health Serv Manage Res 2002; 15: 116-25.

16. Forrest CB, Nutting P, Werner JJ, Starfield B, von Schrader S, Rohde C. Managed health plan effects on the specialty referral process: results from the Ambulatory Sentinel Practice Network referral study. Med Care 2003; 41: 242-53.

17. López-Domínguez JM, Rojas-Marcos I, Sanz-Fernández G, BlancoOllero A, Robledo-Strauss A, Díaz-Espejo C. A descriptive analysis of the demand for ambulatory neurological care in the health district of Huelva. Rev Neurol 2007; 44: 527-30.
18. Alberdi-Ordiozola JC, Sáenz-Bajo N. Factors affecting primary care referrals to specialised care in the Community of Madrid. Aten Primaria 2006; 37: 253-7.

19. Rodríguez Alcalá, FJ, Chacón Fuertes J, Esteban Tudela M, Valles Fernández N, López de Castro F, Sánchez Ramiro A. Motivos de interconsulta entre atención primaria y el segundo nivel. Aten primaria 2005; 36: 137-43.

20. Chan BT, Austin PC. Patient, physician, and community factors affecting referrals to specialists in Ontario, Canada: a population-based, multi-level modelling approach. Med Care 2003; 41: 500-11.

21. Casabella B, Pérez J. Nivel de conocimientos neurológicos del médico general de Atención Primaria. Med Clin (Barc) 1995; 105: 367-369.

22. Lamberts H, Wood M, Hofmans-Okkes IM (eds). The International Classification of Primary Care in the European Community. With a multi-language layer. Oxford: Oxford University Press, 1993.

23. Ortega Tallón MA, Roca Figueres G, Iglesias Rodríguez M, Jurado Serrano JM. Patients over-using a primary care centre: their social, demographic and clinical characteristics and their use of health service facilities. Aten Primaria 2004; 33: 78-85.

24. Weiner J, Starfield B, Stuart M, Powe N, Steinwachs D. Ambulatory care practice variation within a medicaid program. Health Serv Res 1996; 30: 751-70.

25. Forrest CB, Majeed A, Weiner JP, Carroll K, Andrew B Bindman. Comparison of specialty referral rates in the United Kingdom and the United States: retrospective cohort analysis. BMJ 2002; 325: 370-1

26. Grimshaw JM, Winkens RA, Shirran L, Cunningham C, Mayhew A, Thomas R, Fraser C. Interventions to improve outpatient referrals from primary care to secondary care. Cochrane Database Syst Rev 2005; 20 : CD005471.

27. Huerta-Villanueva M, Baiges-Octavio JJ, Martín-Ozaeta G, MuñozFarjas E, Rubio-Borrego F. Evolution of the demand for ambulatory neurological care and pathologies attended in neurology clinics in the health care district of Tortosa, Tarragona. Rev Neurol 2005; 41: 68-74.

28. Sempere AP, Mola S, Medrano V, Esguevillas T, Costa C, Salazar V, Flores-Ruiz JJ, Custardoy J. Descriptive epidemiology of ambulatory neurological care in the Vega Baja (Alicante) area. Rev Neurol 2002; 35: 822-6.

29. Gené-Badia J. Possibilities of routine data bases. Aten Primaria 2006; 37: 258-9.

30. Sackett D, Rosenberg W, Gray J, Haynes Rb. Richardson WS. Evidence based medicine: what it is and what it isn't. BMJ 1996; 312: 71-72.

31. Sánchez-Migallón MJ, Galiano M, Fernández Y. La cefalea en la consulta de neurología general. Neurología 2000; 15: 274-7.

32. Bermejo-Pareja F. Neurology and neurologists in Spain: the consolidation of the family neurologist?. Neurologia 2007; 22: 221-35.

33. Orueta JF, Urraca J, Berraondo I, Darpon J, Aurrekoetxea JJ. Adjusted Clinical Groups (ACGs) explain the utilization of primary care in Spain based on information registered in the medical records: a cross-sectional study. Health Policy 2006; 76: 38-48

34. Sullivan CO, Omar RZ, Ambler G, Majeed A. Case-mix and variation in specialist referrals in general practice. Br J Gen Pract 2005; 55: 52933.

35. Meenan RT, Goodman MJ, Fishman PA, Hornbrook MC, O'KeeffeRosetti MC, Bachman DJ. Using risk-adjustment models to identify high-cost risks. Med Care 2003; 41: 1301-12. 